\title{
ELABORAÇÃO E ACEITABILIDADE DE PRODUTOS DE PANIFICAÇÃO ENRIQUECIDOS COM SEMENTE DE GOIABA (Psidium guajava L.) EM PÓ
}

\author{
A. M. A. UCHÔA THOMAZ ${ }^{1 *}$, E. C. SOUSA ${ }^{1}$, A. LIMA ${ }^{1}$, R. M. T. LIMA ${ }^{1}$, P. A. P. FREITAS ${ }^{1}$, M. A. M. SOUZA ${ }^{1}$, J. C. A. \\ THOMAZ1 e J. O. B. CARIOCA ${ }^{2}$ \\ ${ }^{1}$ Instituto Federal de Educação, Ciência e Tecnologia do Piauí - IFPI \\ ${ }^{2}$ Universidade Federal do Ceará - UFC \\ anamaria.uchoa@gmail.com*
}

Artigo submetido em janeiro/2014 e aceito em outubro/2014

DOI: 10.15628/holos.2014.1895

\section{RESUMO}

A indústria de alimentos, em especial a de processamento de frutos, produz uma grande quantidade de resíduos agroindustriais. Esses resíduos constituem $65-70 \%$ da massa total dos frutos, conforme a espécie do fruto. Uma alternativa que vem crescendo, consiste no aproveitamento destes resíduos como matéria-prima para a produção de alimentos que sejam incluídos na alimentação humana. Este trabalho teve como objetivo propor a elaboração de produtos de panificação com substituições parciais da farinha de trigo por sementes de goiaba em pó. Foram utilizadas três formulações com diferentes percentuais de substituição da farinha de trigo por sementes de goiaba em pó, com níveis de substituição de 5\%, 10\% e $0 \%$ (controle). Como resultado pode-se observar que a preparação bolo doce, formulações com $5 \%$ e $10 \%$ de substituição, apresentaram médias equivalentes aos termos hedônicos "gostei moderadamente" e "gostei muito". A preparação pizza sabor portuguesa, formulação com $5 \%$ de substituição obteve as maiores médias em todos os atributos pesquisadas, cujas médias equivaleram ao termo hedônico "gostei moderadamente". Com isso, pode-se concluir que a adição de pós obtidos de resíduos de frutos como a goiaba é uma alternativa bastante viável para evitar o desperdício e agregar benefícios nutricionais aos produtos de panificação.

PALAVRAS-CHAVE: semente de goiaba (Psidium guajava L.) em pó, desenvolvimento de produtos, análise sensorial.

\section{PREPARATION AND ACCEPTABILITY OF BAKING PRODUCTS ENRICHED WITH GUAVA SEEDS (Psidium guajava L.) POWDER}

\begin{abstract}
The food industry, in particular fruit processing, produces a large amount of waste. These residues constitute 65-70 $\%$ of the total weight of the fruit, depending on the species. An increasingly popular alternative is to make use of this waste as feedstock for the production of foods for human consumption. This work proposes the preparation of bakery products, partially replacing wheat flour by guava seeds powder. Three formulations were used, with three different percentages of replacement of wheat flour by guava seeds powder at levels of $5 \%$ substitution, $10 \%$ and $0 \%$ (control). The results show that
\end{abstract}

the preparation sweet cake formulations with $5 \%$ and $10 \%$ substitution, showed equivalent to medium hedonic terms "like moderately" and "liked". The preparation portuguese pizza flavor formulation with $5 \%$ substitution had the highest mean scores in all the surveyed attributes, equivalent to the hedonic term "like moderately". Thus, we can conclude that the addition of powder made from the residue of fruits like guava is a highly feasible alternative which both avoids wastage and adds nutritional benefits to bakery products.

KEYWORDS: guava seed (Psidium guajava L.) powder, product development, sensory evaluation 


\section{INTRODUÇÃO}

O Brasil é um dos maiores produtores agrícolas mundiais e vem tornando nos últimos anos, uma grande potência no beneficiamento de sua produção. Produtos que antes eram exportados in natura, hoje, passam por diversos processos de industrialização. Em consequência, a agroindústria transformou-se em importante segmento da economia do país (SOUSA, 2009).

A goiaba é um fruto considerado muito importante dentro do contexto da fruticultura brasileira. Sua produção no Brasil está concentrada nos meses de janeiro a março. $O$ aumento no consumo deste fruto está associado à grande divulgação de suas qualidades nutricionais (ZANATTA et al., 2006). Existem aproximadamente trezentas e vinte e quatro espécies de goiaba conhecidas nas regiões tropicais da América Central e América do Sul, distribuídas e cultivadas principalmente em países subtropicais e tropicais. Do gênero Psidium as variedades mais difundidas são a Paluma, Pedro Sato, Ogawa e Kumagai (LIMA et al., 2010). Ela possui características de um fruto com sabor e perfume agradável, e é considerado um fruto altamente nutritivo, pois contém níveis elevados de ácido ascórbico e vários carotenóides tais como o $\beta$-caroteno, rubixantina, luteína, criptoflavina, fitoflueno e licopeno (MERCADANTE, 1999; BRITO et al., 2009).

A indústria de alimentos, em especial a de processamento de frutos, produz ao longo de sua cadeia produtiva uma grande quantidade de resíduos agroindustriais, além de inúmeros problemas ambientais. Essa quantidade de resíduos, segundo alguns autores, constitui $65-70 \%$ da massa total dos frutos, com algumas variações, conforme a espécie do fruto (SOUSA et al., 2011).

No Brasil são processadas cerca de 200 mil ton/ano de goiaba, gerando um resíduo próximo a 12 mil ton/ano, correspondente à semente. Embora os dados sobre a composição química e propriedades funcionais da semente de goiaba sejam escassos, os poucos trabalhos na literatura indicam um conteúdo de óleo entre $8-16 \%$, fibras $50-60 \%$ e proteínas $7,6-9,8 \%$, sendo estes valores variáveis em função de variedade, processamento e condições de cultura (FONTANARI et al., 2007; ROBERTO, 2012).

O desperdício de alimentos é um dos graves problemas que a agricultura mundial enfrenta. De acordo com a Organização das Nações Unidas para Agricultura e Alimentação (FAO-ONU), mais da metade da produção de frutas e verduras é desperdiçada na América Latina. Cerca de $20 \%$ da produção é jogada no lixo antes de sair da propriedade rural (ANUÁRIO BRASILEIRO DE FRUTICULTURA, 2013).

Nos últimos anos, vários pesquisadores brasileiros vêm estudando o aproveitamento de resíduos, como as cascas e sementes de frutos, gerados pelas agroindústrias para a produção de alimentos ou ingredientes. Uma alternativa que vem crescendo desde o início da década de 1970 consiste no aproveitamento destes resíduos como matéria-prima para a produção de alguns alimentos perfeitamente passíveis de serem incluídos na alimentação humana. Assim a utilização econômica de resíduos de frutos oriundos do mercado in natura ou das agroindústrias, aliada ao desenvolvimento de tecnologias para minimizar as perdas nos processos produtivos, podem contribuir de forma significativa para a economia do país e a diminuição dos impactos ambientais (ISHIMOTO et al., 2007; PIOVESANA; BUENO; KLAJN, 2013).

O desenvolvimento de novos produtos acentua a necessidade de testes seguros, eficientes e representativos da opinião do consumidor, tanto quanto o estudo contínuo das mudanças nos 
hábitos alimentares. Os testes de preferência e aceitação com equipes de consumidores são indicados para avaliar, em termos de qualidade hedônica e aceitação, os novos produtos lançados no comércio (TREPTOW et al., 1998; PESSOA et al., 2011).

Sendo assim, o presente trabalho teve como objetivo propor a elaboração de produtos de panificação com substituições parciais da farinha de trigo por sementes de goiaba em pó, bem como verificar a aceitabilidade sensorial e a intenção de compra.

\section{MATERIAIS E MÉTODOS}

\subsection{OBTENÇÃO DOS RESÍDUOS, PROCESSAMENTO E ELABORAÇÃO DO PÓ DAS SEMENTES DE GOIABA.}

Os resíduos agroindustriais de goiaba vermelha (Psidium guajava) cv. Paluma foram cedidos por uma indústria produtora de polpa de frutas congelada (NUTRI VITA), localizada na cidade de Teresina - Piauí. Foram coletadas amostras em diferentes datas de produção (abril de 2011 e maio de 2012). Os resíduos foram retirados diretamente da linha de produção, logo após o processamento da polpa de fruta congelada de goiaba, e transportados imediatamente em caixa isotérmica para o Laboratório de Alimentos do Instituto Federal de Ciência e Tecnologia do Piauí IFPI. Ao chegarem foram armazenados, em sacos plásticos de polietileno sob congelamento à uma temperatura de $-18^{\circ} \mathrm{C}$. Para o início das análises estes resíduos foram descongelados à temperatura média de $25^{\circ} \mathrm{C}$ para serem desidratados em estufa com circulação de ar (TECNAL-modelo TE 394/I), em uma temperatura de $60^{\circ} \mathrm{C}$ por um período de 16 horas. Após a desidratação, os resíduos foram triturados no liquidificador da marca Walita e peneirados.

Para determinar o tamanho das partículas (classificação granulométrica) utilizou-se um conjunto de peneiras com 10, 30, 40, 60, 80, 100, 200 "mesh Tyler" (abertura de 2; 0,60; 0,42; 0,25; 0,$18 ; 0,15 ; 0,075 \mathrm{~mm}$, respectivamente) e a base. Em seguida, as quantidades retidas em cada peneira e na base foram pesadas. Posteriormente a semente de goiaba em pó obtidas dos resíduos da polpa de fruta de goiaba foi acondicionada em frascos tampados de material plástico e cor âmbar previamente higienizados, e armazenados em temperatura de refrigeração, conforme figura 1.

\subsection{FORMULAÇÃO DOS PRODUTOS (BOLO DOCE E PIZZA PORTUGUESA)}

Foram elaborados dois produtos de panificação, bolo doce (Preparação A) e pizza sabor portuguesa (Preparação B). Na elaboração dos bolos foram utilizados farinha de trigo, leite, ovo, manteiga, açúcar refinado, fermento químico e farinha de resíduos de goiaba. Para o preparo da massa da pizza sabor portuguesa utilizou-se farinha de trigo, açúcar refinado, fermento biológico, óleo de soja, ovo, água, sal e farinha de resíduos de goiaba, e para o recheio utilizou-se queijo mussarela, presunto de peru, linguiça calabresa, ovo e molho de tomate. As quantidades dos ingredientes das preparações A e B estão descritas nas tabelas 1 e 2 . Todos os ingredientes foram obtidos no comércio da cidade de Teresina, Piauí. 


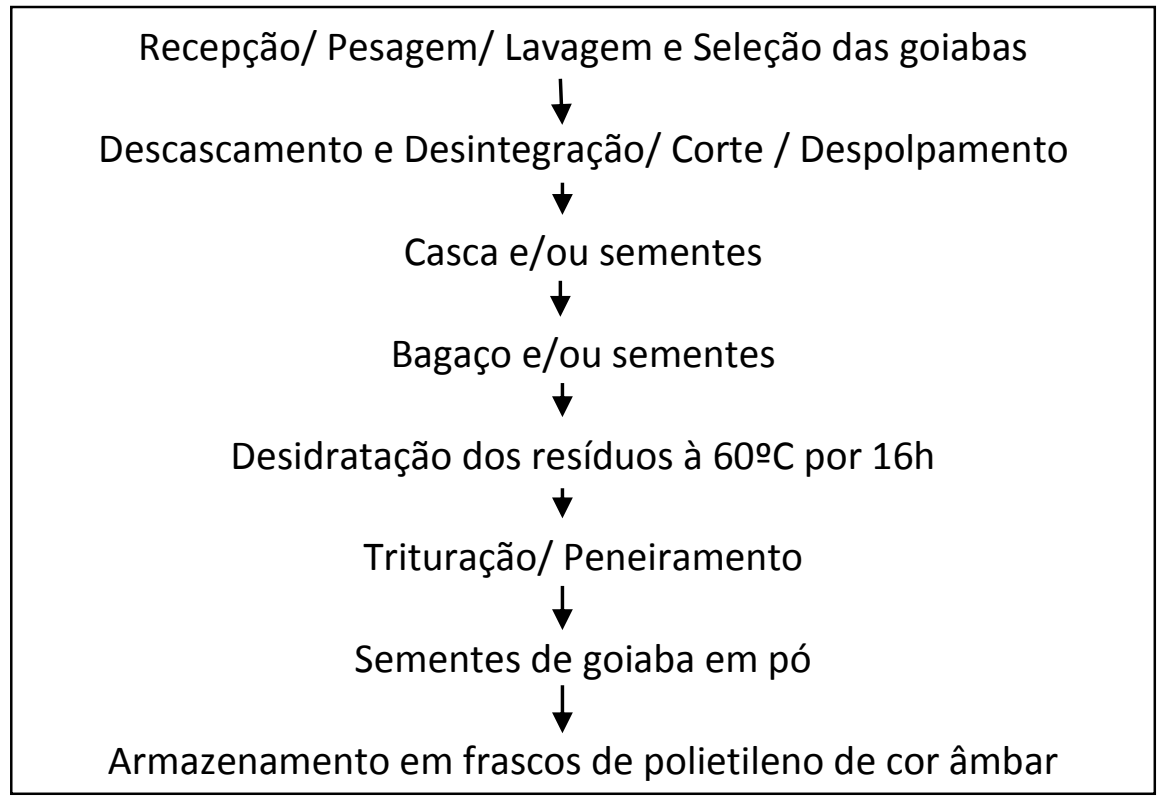

Figura 1: Fluxograma de obtenção das sementes de goiaba e da elaboração da semente de goiaba em pó.

Tabela 1: Formulação do bolo doce enriquecido com diferentes percentuais da semente de goiaba em pó. Teresina, outubro/2012.

\begin{tabular}{c|c|c|c}
\hline \multirow{2}{*}{ Ingrediente } & \multicolumn{2}{|c}{ Quantidade $(\mathrm{g} / \mathrm{mL})$ de acordo com o percentual de substituição } \\
\cline { 2 - 4 } & $\mathrm{FA}$ & $\mathrm{FA}_{5}$ & 324 \\
\hline Farinha de trigo & 360 & 342 & 36 \\
\hline Semente de goiaba em pó & - & 18 & 300 \\
\hline Leite & 300 & 300 & 150 \\
\hline Ovo & 150 & 150 & 120 \\
\hline Manteiga & 120 & 120 & 260 \\
\hline Açúcar refinado & 260 & 260 & 25 \\
\hline Fermento químico & 25 & 25 &
\end{tabular}

As formulações com diferentes percentuais de substituição da farinha de trigo foram denominadas $\mathrm{FA}_{5}(5 \%)$ e $F \mathrm{~A}_{10}(10 \%)$, para preparação $\mathrm{A}$, e $\mathrm{FB} 5(5 \%)$ e $F \mathrm{~B}_{10}(10 \%)$, para preparação $\mathrm{B}$. Foram denominadas $\mathrm{FA}_{0}$ e $\mathrm{FB}_{0}$ os controle (sem substituição da farinha de trigo), para as preparações $A$ e $B$, respectivamente. Para o preparo das formulações, inicialmente, as farinhas foram misturadas (mix) e homogeneizadas nas proporções, denominadas farinha mista.

Tabela 2: Formulação da pizza sabor portuguesa enriquecido com diferentes percentuais da semente de goiaba em pó. Teresina, outubro/2012.

\begin{tabular}{|c|c|c|c|c|}
\hline \multirow{2}{*}{ Etapa de preparo } & \multirow{2}{*}{ Ingrediente } & \multicolumn{3}{|c|}{$\begin{array}{l}\text { Quantidade }(\mathrm{g} / \mathrm{ml}) \text { de acordo com o percentual de } \\
\text { substituição }\end{array}$} \\
\hline & & $\mathrm{FB}_{0}$ & $\mathrm{FB}_{5}$ & $\mathrm{FB}_{10}$ \\
\hline \multirow{6}{*}{ Massa } & Farinha de trigo & 120 & 114 & 108 \\
\hline & $\begin{array}{l}\text { Semente de goiaba em } \\
\text { pó }\end{array}$ & - & 6 & 12 \\
\hline & Fermento biológico & 2,8 & 2,8 & 2,8 \\
\hline & Açúcar refinado & 6,5 & 6,5 & 6,5 \\
\hline & Óleo de soja & 12 & 12 & 12 \\
\hline & Ovo & 6 & 6 & 6 \\
\hline
\end{tabular}




\begin{tabular}{|c|c|c|c|c|}
\hline & Sal & 1,2 & 1,2 & 1,2 \\
\hline & Água & 100 & 100 & 100 \\
\hline \multirow{5}{*}{ Recheio } & Queijo mussarela & 150 & 150 & 150 \\
\hline & Presunto de peru & 150 & 150 & 150 \\
\hline & Linguiça calabresa & 100 & 100 & 100 \\
\hline & Ovo & 100 & 100 & 100 \\
\hline & Molho de tomate & 175 & 175 & 175 \\
\hline
\end{tabular}

Fonte: Laboratório de Análise de Alimentos, IFPI.

Inicialmente, todos os ingredientes das formulações, foram pesados em uma balança digital (Filizola ${ }^{\circledR}$ Platina, Brasil) com precisão de $0,1 \mathrm{~g}$ e capacidade máxima de $15 \mathrm{~kg}$, no Laboratório de Panificação do Instituto Federal de Educação, Ciência e Tecnologia do Piauí - IFPI. Para o preparo dos bolos, primeiramente, bateu-se a manteiga gelada com o açúcar durante 4 minutos (modelo Planetária da marca ARNO), em batedor de globo e velocidade média. Em seguida, foram incorporadas as gemas. Logo após, incorporou-se a farinha mista e o leite em velocidade baixa, até se obter uma massa lisa e homogênea, a mistura foi reservada por alguns minutos. Bateu-se as claras em neve, velocidade alta, com batedor de globo, durante 2 minutos. Com auxílio de uma espátula, seguiu-se a incorporação das claras à massa, por meio de movimentos lentos e circulares. Por fim, foi incorporado o fermento químico. A massa do bolo foi, então, depositada em fôrma de alumínio circular com furo no meio (ROCHEDO, n.o 24), previamente untada com manteiga e polvilhada com farinha de trigo. Os bolos foram assados a $165^{\circ} \mathrm{C}$, em forno de convecção combinado (Perfect Coral 4.0, 157 BRE - 126 A), durante 40 minutos. Os bolos foram então resfriados sobre uma mesa à temperatura ambiente até o momento das análises.

Para elaboração da pizza sabor portuguesa, primeiramente misturou-se os ingredientes água morna e fermento, posteriormente foram deixados em repouso por 10 minutos. Em seguida, adicionou-se açúcar, sal, ovos, óleo de soja, e a farinha mista. Os ingredientes foram misturados manualmente até formar uma massa homogênea, que permaneceu em repouso por 40 minutos. Logo após, as massas foram abertas em forma circular com o auxílio de um rolo, sendo que a espessura aproximada das formulações foi de $0,7 \mathrm{~cm}$. Logo em seguida, as amostras foram assadas em forno (Brastemp ${ }^{\circledR}$ Clean, Brasil), na temperatura de $200{ }^{\circ} \mathrm{C}$, por aproximadamente 15 minutos. Adicionou-se os ingredientes do recheio e colocou-se sob aquecimento por mais 5 minutos.

\subsection{ANÁLISE SENSORIAL E INTENÇÃO DE COMPRA}

Para a realização da análise sensorial utilizou-se o teste efetivo de aceitação global e o teste de intenção de compra nas três formulações $(0,5$ e 10\%) das preparações $A$ e $B$, conforme figura 2 . 


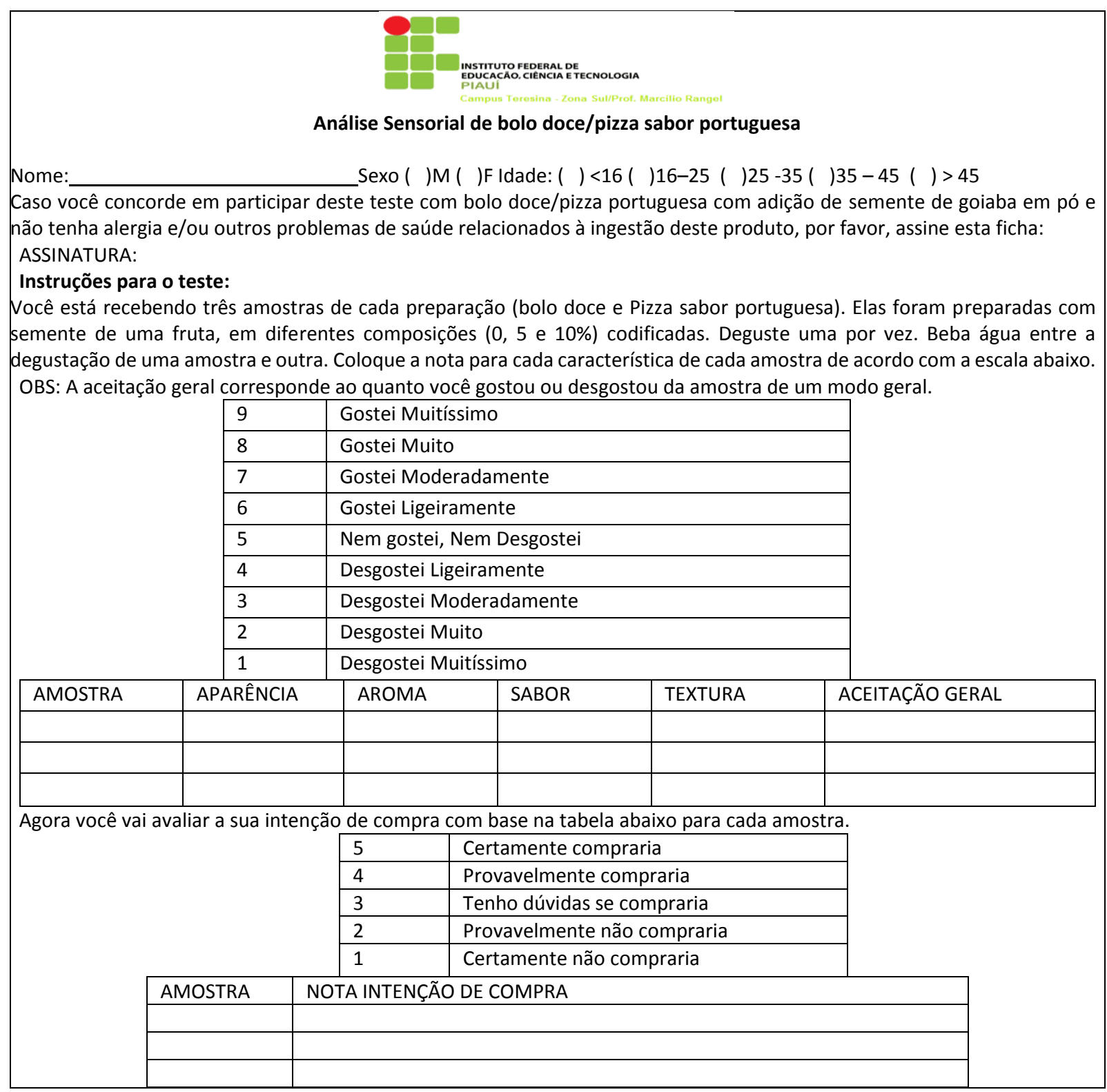

Figura 02: Ficha de avaliação sensorial de bolo doce e pizza sabor portuguesa utilizando a escala hedônica para diversos atributos e a escala de intenção de compra.

Os testes foram realizados com 50 provadores não treinados, de ambos o sexo, com idade entre 16 e 46 anos, selecionados de forma aleatória. Para realização das análises os provadores receberam as informações necessárias para esclarecimento da proposta do trabalho e como deveriam realizar a avaliação do teste. Para execução dos testes as amostras foram apresentadas aos provadores de forma monádica sequencial, cortadas em pedaços de aproximadamente $3 \mathrm{~cm}^{2}$, as quais foram depositadas em recipientes descartáveis codificados com três dígitos aleatórios e dispostas em ordens diferentes para cada provador. Juntamente com as mostras, foi servido um copo com água, para ser degustado entre cada prova, de forma a minimizar o sabor entre uma amostra e outra.

Os provadores utilizaram para avaliar a aceitação global, considerando os atributos de aparência, aroma, sabor, textura, e impressão geral, a escala hedônica estruturada em 9 pontos com valores variando de 1 (desgostei muitíssimo), 5 (não gostei, nem desgostei) e 9 (gostei muitíssimo), conforme descrito por Peryam e Pilgrim (1957). Para a intenção de compra foi 
adotado uma escala hedônica estruturada de 5 pontos, em que 1 representa a pontuação mínima (certamente não compraria) e 5 a maior pontuação (certamente compraria) (SILVA, 1997), conforme figura 2. $O$ índice de aceitabilidade (IA) de cada preparação foi calculado pela expressão: $\mathrm{IA}(\%)=\mathrm{A} \times 100 / \mathrm{B}$, onde $\mathrm{A}=$ nota média obtida para o produto e $\mathrm{B}=$ nota máxima dada ao produto (TEIXEIRA et al., 1987).

\subsection{ANÁLISE ESTATÍSTICA}

Todos os resultados foram apresentados como média $(n=3) \pm$ desvio padrão (DP) e analisados estatisticamente por análise de variância (ANOVA) e teste de Tukey para comparação de médias a $5 \%$ de significância $(p<0,05)$, utilizando o programa SAS ${ }^{\circledR}$ 'Statistical Analytical Systems' (SAS, 2008) for Windows.

\section{RESULTADOS E DISCUSSÃO}

\subsection{CARACTERIZAÇÃO DA FARINHA}

A partir das sementes obtidas dos resíduos do processamento de polpa congelada de goiaba obteve-se um pó que apresentou após análises com peneiras de 10 a 200 "mesh Tyler", uma granulometria variando entre $0,42 \mathrm{~mm}$ e $0,60 \mathrm{~mm}$ de diâmetro, visto que a retenção de uma maior quantidade de pó se deu nas peneiras com "mesh" entre 30 e 40.

Levando-se em consideração à amostra úmida, pode-se observar um rendimento de aproximadamente 54\%, resultado superior ao encontrado por Melo (2010), que obteve no resíduo de goiaba liofilizado rendimento de 38,32\%. Em outro estudo realizado por Prado (2009), obteve para a polpa de goiaba liofilizada um rendimento de 9,94\%. Em frutos de outras mirtáceas, como araçá-pera (Psidium acutangulum) e araçá-boi (Eugenia stipitata) o rendimento foi de 55,01\% e 63\%, respectivamente (FERREIRA, 1992; REBOUÇAS, 2008). O bom rendimento apresentado pelo resíduo de goiaba pode ser atribuído a grande quantidade de semente na sua composição.

\subsection{ANÁLISE SENSORIAL E INTENÇÃO DE COMPRA}

$O$ primeiro teste sensorial realizado com as amostras teve como objetivo verificar a preferência dos provadores em relação às três formulações de bolo doce, onde estas diferiam entre si pela percentual de farinha de resíduo de goiaba adicionadas a mistura. Os dados obtidos nesta análise podem ser observados na tabela 3.

Tabela 3: Médias do teste sensorial afetivo e intenção de compra realizados para as formulações de bolo doce com $0 \%$ (FA0), adicionado de 5\% (FA5) e 10\% (FA10). Teresina, outubro/2012.

\begin{tabular}{c|c|c|c}
\hline \multirow{2}{*}{ Atributo sensorial } & \multicolumn{3}{|c}{ Bolo doce* } \\
\cline { 2 - 4 } & $\mathrm{FA}_{0}$ & $\mathrm{FA}_{5}$ & $\mathrm{FA}_{10}$ \\
\hline Aparência & $6,57 \pm 0,11^{\mathrm{b}}$ & $7,77 \pm 0,15^{\mathrm{a}}$ & $7,83 \pm 0,20^{\mathrm{b}}$ \\
\hline Aroma & $7,10 \pm 0,10^{\mathrm{b}}$ & $7,70 \pm 0,26^{\mathrm{a}}$ & $8,00 \pm 0,00^{\mathrm{a}}$ \\
\hline Sabor & $7,77 \pm 0,15^{\mathrm{a}}$ & $8,20 \pm 0,26^{\mathrm{a}}$ & $7,80 \pm 0,10^{\mathrm{a}}$ \\
\hline Textura & $7,34 \pm 0,15^{\mathrm{b}}$ & $8,10 \pm 0,10^{\mathrm{a}}$ & $7,93 \pm 0,15^{\mathrm{a}}$ \\
\hline Impressão geral & $7,93 \pm 0,15^{\mathrm{a}}$ & $8,23 \pm 0,25^{\mathrm{a}}$ & $4,10 \pm 0,00^{\mathrm{a}}$ \\
\hline
\end{tabular}

*Médias seguidas pela mesma letra nas linhas não diferem entre si ao nível de $5 \%$ de significância pelo teste de Tukey. Fonte: Laboratório de Análise de Alimentos, IFPI. 
Conforme a tabela 3, para os atributos aparência e aroma as médias encontradas variaram entre 6 e 7, o que corresponde a um nível de preferência na escala hedônica entre "gostei ligeiramente" e "gostei moderadamente". Já nos atributos de sabor, textura e impressão geral as médias encontradas variaram entre 7 e 8 , o que corresponde a um nível de preferência na escala hedônica entre "gostei moderadamente" e "gostei muito".

As avaliações gostei muito (8) e gostei moderadamente (7) foram as mais assinaladas para o atributo sabor, textura e impressão geral. Resultados similares foram relatados por Lima (2007), ao avaliarem o aroma de formulações de pães adicionados de farinha de linhaça e farinha de maracujá. O que também corroboram os resultados obtidos por Martin et al. (2012), que também obtiveram a maioria das respostas dadas pelos provadores para os atributos superiores a 7 , indicando boa aceitação do bolo acrescido do suco de cascas de abacaxi.

Avaliando-se os dados de preferência pela Análise de Variância (ANOVA), para os tributos aparência e aroma, percebeu-se que a amostra $\mathrm{FA}_{5}$, obteve maior média e apresentou diferença significativa, das amostras $\mathrm{FA}_{0}$ e $F A_{10}$, ao nível de significância de $5 \%$. No atributo textura constatou-se que as formulações $F A_{5}$ e $F A_{10}$, apresentaram notas maiores que $F A_{0}(p<0,05)$. Nos atributos sabor e impressão geral, as amostras não apresentaram diferença significativa ao nível de significância de 5\%. Entretanto, todas as amostras permaneceram com resultados acima de $50 \%$, podendo ser consideradas com boa aceitação.

As formulações $F A_{5}$ e $F A_{10}$, apresentaram maiores notas de intenção de compra, 4,33 $\pm 0,15$ e 4,10 $\pm 0,00$, respectivamente, que a formulação $F A_{0}, 3,20 \pm 0,10(p<0,05)$, isso demonstra que os provadores "certamente comprariam" o bolo com substituição de 5 e $10 \%$ da farinha de trigo. Com isso, pode avaliar que é viável a comercialização desse produto com a utilização da semente de goiaba em pó.

Conforme Nassu (2007), os métodos afetivos expressam opinião (preferência ou aceitabilidade) do consumidor potencial de um produto a respeito das características específicas do mesmo. Os dados obtidos no teste afetivo aplicado às três formulações de pizza sabor portuguesa pode ser observado na tabela 4.

Tabela 4: Médias do teste sensorial afetivo e intenção de compra realizados para as formulações de pizza sabor portuguesa com 0\% (FB0), adicionado de 5\% (FB5) e 10\% (FB10) de semente de goiaba em pó. Teresina, outubro/2012.

\begin{tabular}{c|c|c|c}
\hline \multirow{2}{*}{ Atributo sensorial } & \multicolumn{3}{|c}{ Pizza sabor portuguesa* $^{*} \mathrm{FB}_{10}$} \\
\cline { 2 - 4 } & $\mathrm{FB}_{0}$ & $\mathrm{FB} 5$ & $6,60 \pm 0,10^{\mathrm{b}}$ \\
\hline Aparência & $7,10 \pm 0,10^{\mathrm{a}}$ & $7,10 \pm 0,10^{\mathrm{a}}$ & $6,47 \pm 0,30^{\mathrm{a}}$ \\
\hline Aroma & $6,80 \pm 0,40^{\mathrm{a}}$ & $7,10 \pm 0,10^{\mathrm{a}}$ & $6,10 \pm 0,20^{\mathrm{b}}$ \\
\hline Sabor & $6,90 \pm 0,10^{\mathrm{a}}$ & $7,54 \pm 0,50^{\mathrm{a}}$ & $6,57 \pm 0,30^{\mathrm{b}}$ \\
\hline Textura & $6,50 \pm 0,00^{\mathrm{b}}$ & $7,46 \pm 0,35^{\mathrm{a}}$ & $6,73 \pm 0,55^{\mathrm{a}}$ \\
\hline Impressão geral & $6,90 \pm 0,36^{\mathrm{a}}$ & $7,47 \pm 0,15^{\mathrm{a}}$ & $3,20 \pm 0,20^{\mathrm{b}}$ \\
\hline
\end{tabular}

*Médias seguidas pela mesma letra nas linhas não diferem entre si ao nível de $5 \%$ de significância pelo teste de Tukey. Fonte: Laboratório de Análise de Alimentos, IFPI.

$\mathrm{Na}$ tabela 4, verificou-se que a maioria das respostas dadas pelos provadores, para a formulação $\mathrm{FB}_{5}$, para os atributos testados, foram superiores a 7 "gostei moderadamente", indicando boa aceitação da pizza sabor portuguesa com substituição de $5 \%$ da farinha de trigo pela a semente de goiaba em pó. 
Para os atributos aparência e sabor as formulações $\mathrm{FB}_{0}$ e $\mathrm{FB}_{5}$, obtiveram maiores médias e não apresentaram diferença estatística ao nível de $5 \%$ de significância, pelo teste de tukey. Os provadores avaliaram com maior média à formulação $\mathrm{FB}_{5}$, no atributo textura, o que diferiu das demais formulações.

Com relação, aos atributos aroma e impressão geral, observou-se que as amostras controle $\left(\mathrm{FB}_{0}\right)$ e adicionada de $5 \%\left(\mathrm{FB}_{5}\right)$ de semente de goiaba em pó não apresentaram diferença entre si ( $p>0,05)$, porém a formulação $F B_{5}$ apresentou média superior $(7,47 \pm 0,15)$, em relação ao controle, o que representa uma resposta na escala hedônica de "gostei moderadamente". Russo et al. (2012) avaliaram a aceitabilidade sensorial de massa de pizza acrescida de farinhas de trigo integral e de linhaça (Linum usitatissimum L.) entre adolescentes e comprovou que a adição de até 5,0\% de farinha de linhaça em massa de pizza foi melhor aceita pelos provadores dentre aqueles contendo este ingrediente, obtendo-se aceitação sensorial semelhante ao produto padrão.

Para o teste de intenção de compra percebeu-se que o produto elaborado com substituição de $5 \%$ da farinha de trigo pela semente de goiaba em pó obteve uma boa aceitação por parte dos provadores, uma vez que sua média foi de 4,13 $\pm 0,05$ e diferiu estatisticamente das demais médias, indicando que o consumidor "provavelmente compraria", o que pode representar boas vendas deste produto.

Uma análise mais detalhada dos dados gerados pela análise sensorial revelou o grau de aceitação dos bolos doces e pizzas sabor portuguesa acrescidos da semente de goiaba em pó. Os resultados obtidos para a aceitação dos produtos elaborados, tanto da formulação A quanto da formulação B, estão apresentados na figura 3 .

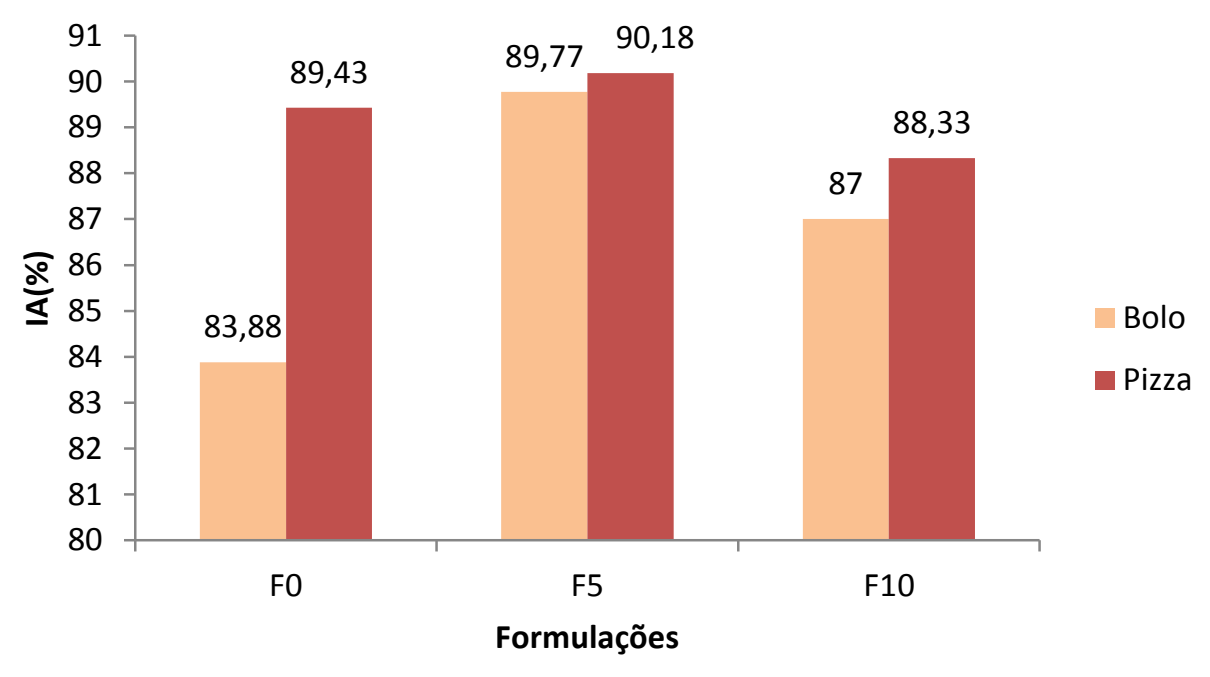

Figura 3: Aceitabilidade quanto aos atributos avaliados no bolo doce e pizza sabor portuguesa. Fo: controle; F5: substituição de $5 \%$ da farinha de trigo; e $F_{10}$ : substituição de $10 \%$ da farinha de trigo.

Segundo Souza (2007), um alimento com mais de 70\% de aprovação indica boa aceitação. Sendo assim, todas as formulações, tanto de bolo doce quanto de pizza sabor portuguesa, obtiveram percentuais acima deste valor. De acordo com a Figura 3, os provadores avaliaram que a formulação que substituiu em $5 \%$ a farinha de trigo pela semente de goiaba em pó, foi a que apresentou maior aceitabilidade, tanto na preparação de bolo doce quanto na de pizza sabor portuguesa, chegando a um percentual de aproximadamente $90 \%$. Medeiros et al. (2012), ao 
analisar sensorialmente trufa de caju obteve um índice de aceitação superior à $50 \%$, chegando a $72 \%$ no atributo textura.

\section{CONCLUSÃO}

Os resultados apresentados permitiram comprovar que a substituição de $5 \%$ e $10 \%$ de farinha de trigo pela semente de goiaba em pó na massa do bolo doce e na pizza sabor portuguesa apresentou melhor aceitabilidade pelos provadores, obtendo-se aceitação sensorial melhor do que ao produto controle. Em geral, a adição da semente de goiaba em pó é uma alternativa viável para evitar o desperdício, agregar valor econômico à produção de polpa congelada de frutas, tendo em vista que esta atividade gera grandes resíduos que ainda são pouco explorados industrialmente, e agregar benefícios nutricionais em produtos de panificação.

O desenvolvimento de novos produtos com boa aceitabilidade por parte dos consumidores e com altas expectativas de aceitação no mercado, como bolo doce e pizza sabor portuguesa, demonstra a possibilidade de reaproveitamento de partes de frutos que eram desperdiçados pela agroindústria de frutos, contribuindo também para a minimização do impacto ao meio ambiente.

\section{AGRADECIMENTOS}

Ao $\mathrm{CNPq}$ /Capes e FAPEPI, pelo apoio financeiro, à industria produtora de polpa de fruta (Nutri Vita), pelo fornecimento dos resíduos de goiaba e ao Instituto Federal de Educação, Ciência e Tecnologia do Piauí (IFPI), pelo apoio institucional.

\section{REFERÊNCIAS BIBLIOGRÁFICAS}

1. ANUÁRIO BRASILEIRO DE FRUTICULTURA. Heloísa Poll...[et al]. Editora Gazeta Santa Cruz Ltda. Santa Cruz do Sul. 136p. 2013.

2. BRITO, C.A.K. de., SIQUEIRA, P.B., SOUZA, J.C. de., BOLINI, H.M.A. In vitro antioxidant capacity, phenolic, ascorbic acid and lycopene content of guava (Psidium guajava I.) juices and nectars. Boletim da CEPPA, Curitiba, v. 27, n. 2, p. 175-182, jul./dez. 2009.

3. FONTANARI, G.G., JACON, M.C., PASTRE, I.A., FERTONANI, F.L., NEVES, V.A., BATISTUTI, J.P. Isolado proteico de semente de goiaba (Psidium guajava): caracterização de propriedades funcionais. Ciência e Tecnologia dos Alimentos, Campinas, v. 27(supl.), p. 73-79, ago. 2007.

4. FERREIRA, S.A.N. Biometria de frutos de araçá-boi (Eugenia stipitata McVaugh). Acta Amazônica, Manaus, v. 22, n. 3, p. 295-302, 1992.

5. ISHIMOTO, F.Y., HARADA, A.I., BRANCO, I.G., CONCEIÇÃO, W.A.S., COUTINHO, M.R. Aproveitamento alternativo da casca do maracujá-amarelo (Passifora edulis $\mathrm{f}$. var. favicarpa Deg.) para produção de biscoitos. Revista Ciências Exatas e Naturais, Guarapuava, v. 9, n. 2, p. 279-292, 2007.

6. LIMA, R.K., CARDOSO, M. das G., ANDRADE, M.A., NASCIMENTO, E.A., MORAIS, S.A.L. de., NELSON, D.L. Composition of the essential oil from the leaves of tree domestic varieties and one wild variety of the guava plant (Psidium guajava L., Myrtaceae). Revista Brasileira de Farmacognosia, São Paulo, v. 20, n. 1, p. 41-44, Jan./Mar. 2010. 
7. LIMA, C.C. Aplicação das farinhas de linhaça (Linum usitatissimum L.) e maracujá (Passifora edulis Sims f. favicarpa Deg.) no processamento de pães com propriedades funcionais. Fortaleza, 2007. Dissertação de mestrado. Universidade Federal do Ceará, 2007.

8. MARTIN, J.G.P., MATTA JÚNIOR, M.D. de., ALMEIDA, M.A. de., SANTOS, T. dos., SPOTO, M.H.F. Avaliação sensorial de bolo com resíduo de casca de abacaxi para suplementação do teor de fibras. Revista Brasileira de Produtos Agroindustriais, Campina Grande, v. 14, n. 3, p. 281-287, 2012.

9. M. J. M. MEDEIROS, M.J.M., SILVA, J.F., FAUSTINO, M.V.S., SANTOS, M.F.G., L. C S. ROCHA, L.C.S., CARNEIRO, L.C. Aceitação sensorial e qualidade microbiológica de trufas de caju obtidas artesanalmente. HOLOS, Natal- RN, Ano 28, v. 2, 2012.

10. MELO, P.B. Composição química e atividade biológica de resíduos agroindustrial. Piracicaba, 2010. Dissertação de Mestrado em Ciências. Escola Superior de Agricultura "Luiz de Queiroz". Universidade de São Paulo, 2010.

11. MERCADANTE, A.Z. Chromatographic separation of carotenoids. Archivos Latinoamericanos de Nutricion, v. 49, p. 52-57, 1999.

12. NASSU, R.T. Análise Sensorial de Carne: Conceitos e Recomendações. São Carlos: Embrapa Pecuária Sudeste, 2007. 7 p. (Comunicado Técnico - Embrapa Pecuária Sudeste, n. 79).

13. PESSOA, T., AMARAL, D.S., DUARTE, M.E.M., CAVALCANTI MATA, M.E.R.M., GURJÃO, F.F. Avaliação sensorial de goiabas passas obtida por técnicas combinadas de desidratação osmótica e secagem. HOLOS, Ano 27, v. 4, p. 137-147, 2011.

14. PIOVESANA, A., BUENO, M.M., KLAJN, V.M. Elaboração e aceitabilidade de biscoitos enriquecidos com aveia e farinha de bagaço de uva. Brazilian Journal of Food Technology, Campinas, v. 16, n. 1, p. 68-72, jan./mar. 2013.

15. PERYAM, D.R., PILGRIM, F.J. Hedonic scale method of measuring food preferences. Food Technology, v. 11, n. 9, p. 9-14, 1957.

16. PRADO, A. Composição fenólica e atividade antioxidante de frutas tropicais. Dissertação de Mestrado em Ciências. Piracicaba, 2009. Escola Superior de Agricultura "Luiz de Queiroz", 2009.

17. REBOUÇAS, E.R., GENTIL, D.F. de O., FERREIRA, S.A. do N. Caracterização física de frutos e sementes de goiaba-da-costa-rica, produzidos em Manaus, Amazonas. Revista Brasileira de Fruticultura, Jaboticabal - SP, v. 30, n. 2, p. 546-548, Junho 2008.

18. ROBERTO, B.S. Resíduo de goiaba: metabolismo em ratos e aplicabilidade em barra de cereais. Santa Maria, 2012. Dissertação de mestrado. Universidade Federal de Santa Maria, Rio Grande do Sul, 2012.

19. RUSSO, C.B., SOSTISSO, C.F., PASQUAL, I.N., NOVELLO, D., DALLA SANTA, H.S., BATISTA, M.G. Aceitabilidade sensorial de massa de pizza acrescida de farinhas de trigo integral e de linhaça (Linum usitatissimum L.) entre adolescentes. Revista do Instituto Adolfo Lutz, v. 71, n. 3, p. 488-94, 2012.

20. SAS (Statistical Analysis System) for Windows, Version 9.2, USA: Microsoft Corporation, 2008. CD-ROM.

21. SILVA, M.A.A.P. Métodos de avaliação sensorial de alimentos. Apostila: Escola de extensão da UNICAMP, 1997, 71p. 
22. SOUSA, B.A.A. Funcionalidade dos extratos fenólicos obtidos pelo cultivo semi-sólido de resíduos de abacaxi (Ananas comosus L.) e goiaba (Psidium guajava L.). Natal, 2009. Dissertação de Mestrado em Engenharia Química, Universidade Federal do Rio Grande do Norte, 2009.

23. SOUSA, C.M. de M., SILVA, H.R., VIEIRA JÚNIOR, G.M., AYRES, M.C.C., SOUSA, M.S.B., VIEIRA, L.M., SILVA, M. de J.M da., LIMA, A de. Caracterização nutricional e compostos antioxidantes em resíduos de polpas de frutas tropicais. Ciência Agrotécnica, Lavras, v. 35, n. 3, p. 554-559, mai./jun., 2011.

24. SOUZA, P.D.J., NOVELLO, D., ALMEIDA, J.M., QUINTILIANO, D.A. Análise sensorial e nutricional de torta salgada elaborada através do aproveitamento alternativo de talos e cascas de hortaliças. Alimento e Nutrição, Araraquara, v. 18, n. 1, p.55-60, 2007.

25. TEIXEIRA, E., MEINERT, E.M., BARBETTA, P.A. Análise sensorial de alimentos. Florianópolis: Ed. UFSC, 1987. 180p.

26. TREPTOW, R.O., QUEIROZ, M.I., ANTUNES, P.L. Preferência e Aceitação de Fatias Desidratadas de Maçãs (Malus domestica Borkh). Revista Brasileira de Agrociência, v. 4, n. 1, p. 41-46, 1998.

27. ZANATTA, C.L., ZOTARELLI, M.F., CLEMENTE, E. Peroxidase (POD) e polifenoloxidase (PPO) em polpa de goiaba (Psidium guajava L.). Ciência e Tecnologia de Alimentos, Campinas, v. 26, n. 3, p. 705-708, jul./set, 2006. 